University of Wollongong

Research Online

Australian Institute for Innovative Materials -

Papers

Australian Institute for Innovative Materials

$1-1-2020$

Stabilization of Skyrmions in a Nanodisk Without an External Magnetic

Field

Hang Li

Collins Akosa

Peng Yan

Yuanxu Wang

Zhenxiang Cheng

University of Wollongong, cheng@uow.edu.au

Follow this and additional works at: https://ro.uow.edu.au/aiimpapers

Part of the Engineering Commons, and the Physical Sciences and Mathematics Commons

Research Online is the open access institutional repository for the University of Wollongong. For further information contact the UOW Library: research-pubs@uow.edu.au 


\title{
Stabilization of Skyrmions in a Nanodisk Without an External Magnetic Field
}

\author{
Abstract \\ We study, in the absence of a magnetic field, the stability of skyrmions in a disk-shaped ferromagnetic \\ multilayer in the presence of locally pinned spins at the edge. This particular boundary condition \\ suppresses the formation of helical states yet enhances the stabilization of states with rotational \\ symmetry, such as Neél skyrmions. Numerical simulation shows that the size of the disk is a viable \\ parameter to control the creation and annihilation of skyrmionic states. We also find that the \\ demagnetization field renormalizes the Dzyaloshinskii-Moriya interaction. This study opens an alternative \\ avenue to the generation, stabilization, and control of magnetic skyrmions in field-free heterostructures.

\section{Disciplines} \\ Engineering | Physical Sciences and Mathematics \\ Publication Details \\ Li, H., Akosa, C. A., Yan, P., Wang, Y. \& Cheng, Z. (2020). Stabilization of Skyrmions in a Nanodisk Without \\ an External Magnetic Field. Physical Review Applied, 13 (3), 034046-1-034046-8.
}




\title{
Stabilization of Skyrmions in a Nanodisk Without an External Magnetic Field
}

\author{
Hang Liø,,${ }^{1, *}$ Collins Ashu Akosa $\odot,{ }^{1,2,3, \dagger}$ Peng Yan, ${ }^{4}$ Yuanxu Wang, ${ }^{1}$ and Zhenxiang Cheng ${ }^{5}$ \\ ${ }^{1}$ Institute for Computational Materials Science, School of Physics and Electronics, Henan University, \\ Kaifeng 475004, China \\ ${ }^{2}$ RIKEN Center for Emergent Matter Science (CEMS), 2-1 Hirosawa, Wako, Saitama 351-0198, Japan \\ ${ }^{3}$ Department of Theoretical and Applied Physics, African University of Science and Technology (AUST), \\ Km 10 Airport Road, Galadimawa, Abuja F.C.T, Nigeria \\ ${ }^{4}$ School of Electronic Science and Engineering and State Key Laboratory of Electronic Thin Films and Integrated \\ Devices, University of Electronic Science and Technology of China, Chengdu 610054, China \\ ${ }^{5}$ Institute for Superconducting \& Electronic Materials, Australian Institute of Innovative Materials, University of \\ Wollongong, Innovation Campus, Squires Way, North Wollongong, NSW 2500, Australia
}

(Received 20 May 2019; revised manuscript received 10 January 2020; accepted 3 March 2020; published 18 March 2020)

\begin{abstract}
We study, in the absence of a magnetic field, the stability of skyrmions in a disk-shaped ferromagnetic multilayer in the presence of locally pinned spins at the edge. This particular boundary condition suppresses the formation of helical states yet enhances the stabilization of states with rotational symmetry, such as Néel skyrmions. Numerical simulation shows that the size of the disk is a viable parameter to control the creation and annihilation of skyrmionic states. We also find that the demagnetization field renormalizes the Dzyaloshinskii-Moriya interaction. This study opens an alternative avenue to the generation, stabilization, and control of magnetic skyrmions in field-free heterostructures.
\end{abstract}

DOI: 10.1103/PhysRevApplied.13.034046

\section{INTRODUCTION}

Spin-orbit interaction [1,2], by coupling electron spin to its orbital angular momentum, drives a variety of phenomena including the spin Hall effect, chiral magnonics and, more recently, Majorana fermions in condensed-matter systems [3-6]. In a noncentrosymmetric crystal [7-9], such as B20 magnets or a system lacking inversion symmetry, such as heavy metal and/or ferromagnetic heterostructures, bulk or interfacial spin-orbit coupling induces a chiral antisymmetric exchange interaction. This is the Dzyaloshinskii-Moriya interaction (DMI) [10,11]. Given two spins $\mathbf{S}_{i}$ and $\mathbf{S}_{j}$ on neighboring sites $i$ and $j$, the DMI can be expressed as

$$
H_{\mathrm{DMI}}=-\sum_{i j} \mathbf{D}_{i j} \cdot\left(\mathbf{S}_{i} \times \mathbf{S}_{j}\right)
$$

Parameter $\mathbf{D}_{i j}$ is perpendicular to the vector connecting two neighboring magnetic moments and its magnitude characterizes the interaction strength. In a chiral magnet, the DMI lowers the system energy by forming magnetic skyrmions, a swirling spin texture at the nanoscale. This particlelike topological excitation, together with its unique

\footnotetext{
*hang.li@vip.henu.edu.cn

†collins.akosa@riken.jp
}

real-space characteristics, have attracted much attention [12-21].

Stabilization of skyrmions in the host material is instrumental to spintronic applications [22-24]. In a recent experiment [25], a combination of the DMI and exchange interaction gives rise to a periodic spin helical state in helimagnets. This state is further tailored into individual skyrmionic states by using, for instance, external magnetic fields [15-18], electric fields [19], or geometric confinement $[13,20,21]$. In the presence of a magnetic field, geometric structures such as the nanodisk can accommodate stable skyrmionic states $[8,9,26]$. This, from the energy point of view, is an outcome of two competing effects. On the one hand, the local energy density decays from the center of the nanodisk toward the edge [27]. At the edge, the tilted spins hold the same or opposite chirality as the skyrmion, creating a barrier that limits the radius of the skyrmion [26]. On the other hand, the magnetostatic energy favors a swirling spin structure; hence the total energy is lowered. A recent report has shown that in $\mathrm{Fe}-\mathrm{Ge}$, a skyrmionic state can survive in the absence of a magnetic field [28]. In this experiment, in addition to a skyrmionic state, the spin texture consists of a ring-shaped vortex and a ferromagnetic strip. The vortex stems from the DMI acting at the edge and is independent of the magnetic field [29]. Yet the ferromagnetic strip is driven by the ferromagnetic order at low temperature [30]. 
In this paper, we explore the viability of stabilizing skyrmionic states in the absence of external fields. We show that, by imposing locally pinned spins at the edge, small-size skyrmionic states can be nucleated in a thin-film nanodisk. In such a constrained system with this particular boundary condition, we may generate stable magnetic skyrmions and other rich spin textures in a broad range of geometric sizes and DMI magnitudes.

\section{THEORETICAL MODEL}

A chiral spin spiral due to the DMI may exist at an interface between two ferromagnetic regions with opposite magnetizations [31-34]. The exchange and the DMI are not the only interactions that determine the spin texture; the geometry of the boundary matters too. In a finite system with a strong DMI, the spins may rotate up to $2 \pi$ across two antiparallel ferromagnetic regions [35]. From the symmetry point of view, the skyrmion can be viewed as a $2 \pi$-phase domain wall with a $\pi$ rotation around its midpoint in the plane. In this picture, if the DMI is sufficiently large and the preferred axis of the spin helix is constrained by the spins at the edge, we expect the spin spiral to be driven into an ordered skyrmionic state with a spin rotation of $2 \pi$, even without an antiparallel ferromagnetic region.

Consider the model system sketched in Figs. 1(b) and 1(c). It consists of an ultrathin nanodisk (gray) deposited on top of a ferromagnet (blue). We may choose, for example, $\mathrm{Co} / \mathrm{Pt}$ or $\mathrm{CoFeB} / \mathrm{Ta}$ to integrate both the $\mathrm{DMI}$ and exchange couplings. Furthermore, a pinning layer (black) is deposited around the nanodisk, as shown in Fig. 1(b). The ring-shaped pinning layer may be realized by the

(a)

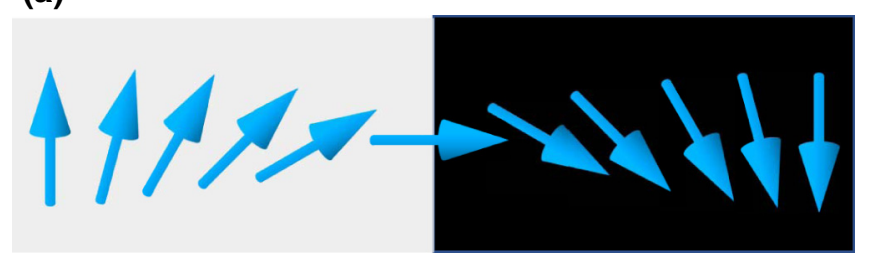

(b)

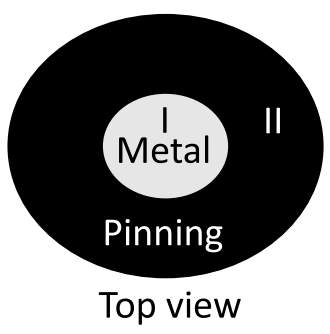

(c)

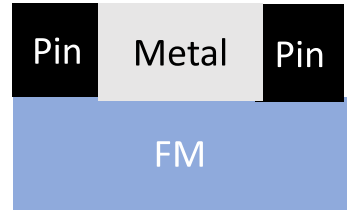

Side view

FIG. 1. (a) A schematic view of the magnetization vector of a domain wall between two ferromagnetic media with opposite magnetizations. (b) Top and (c) side views of a nanodisk that is an ultrathin bilayer film. A pinning layer (area II, in black) is deposited on a ferromagnetic film (blue) to wrap around the metal film (area I, in gray).

deposition of heavy metals (such as $\mathrm{Hf}$ ), metallic oxides (such as $\mathrm{MgO}$ or $\mathrm{Al}_{2} \mathrm{O}_{3}$ ) [33], or permanent magnetic nanoparticles [36-38]. We assume an ideal situation in which the magnetization of the bottom ferromagnet is coerced in a specific direction. In this case, we introduce, within the ferromagnet, locally pinned spins that interact with the free spins in the central region and play the role of a spin boundary condition. Possible skyrmion nucleation may take place in the central region that is directly underneath the metal.

\section{MICROMAGNETIC SIMULATION}

We conduct a comparative study on two setups to ascertain the role of the pinned spins in stabilizing the skyrmions. The first setup is a nanodisk without the ringshaped pinning layer, which is often referred to throughout the rest of paper as a free boundary or free spins. The second setup is with the pinning layer and is referred to as a fixed boundary or pinned spins.

Within the micromagnetism framework, the magnetization dynamics of the $i$ th magnetic unit cell $\mathbf{M}^{(i)}$ are governed by the Landau-Lifshitz-Gilbert (LLG) equation

$$
\frac{d \mathbf{M}^{(i)}}{d t}=-\gamma \mathbf{M}^{(i)} \times \mathbf{H}_{\mathrm{eff}}^{(i)}+\frac{\alpha}{M_{s}} \mathbf{M}^{(i)} \times \frac{d \mathbf{M}^{(i)}}{d t},
$$

where $\gamma$ is the gyromagnetic ratio, $\alpha$ is the Gilbert damping constant, and $M_{s}$ is the saturation magnetization. In Eq. (2), the effective field of the $i$ th unit cell can be derived from $\mathbf{H}_{\text {eff }}^{(i)}=-\partial \mathcal{E} /\left(\mu_{0} \partial \mathbf{M}^{(i)}\right)$, where $\mu_{0}$ is the permeability of free space and $\mathcal{E}$ is the magnetic energy density that incorporates exchange, anisotropy, magnetostatic energy, and the DMI. In our model, the effective field is

$$
\begin{aligned}
\mathbf{H}_{\mathrm{eff}}^{(i)} & =\frac{2 A_{\mathrm{ex}}}{\mu_{0} M_{s}^{2}} \nabla^{2} \mathbf{M}^{(i)}+\frac{2 K_{u}}{\mu_{0} M_{s}^{2}}\left[\mathbf{M}^{(i)} \cdot \hat{\mathbf{z}}\right] \hat{\mathbf{z}} \\
& +\frac{2 D}{\mu_{0} M_{s}^{2}}\left[\frac{\partial M_{z}^{(i)}}{\partial x} \hat{\mathbf{x}}+\frac{\partial M_{z}^{(i)}}{\partial y} \hat{\mathbf{y}}-\left(\frac{\partial M_{x}^{(i)}}{\partial x}+\frac{\partial M_{y}^{(i)}}{\partial y}\right) \hat{\mathbf{z}}\right] \\
& +\sum_{j} \mathcal{K}_{i j} \mathbf{M}^{(j)} .
\end{aligned}
$$

Here, $A_{\text {ex }}$ denotes the exchange stiffness [39], $K_{u}$ is the uniaxial anisotropy constant, $\hat{\mathbf{x}}, \hat{\mathbf{y}}, \hat{\mathbf{z}}$ are the unit directional vectors, $\mathcal{K}_{i j}$ is the demagnetizing tensor [40-42], and $D$ quantifies the strength of the DMI [43]. Even though one would expect the distribution of the DMI to be inhomogeneous along the radial direction due to material variation, we assume that the pinning effect at the edge is so strong that the DMI in this region can be neglected. We also neglect the dipole-dipole interaction since it becomes local in the limit of zero thickness [44]. The micromagnetic simulations are performed using the MuMax3 package [40]. 
(a)

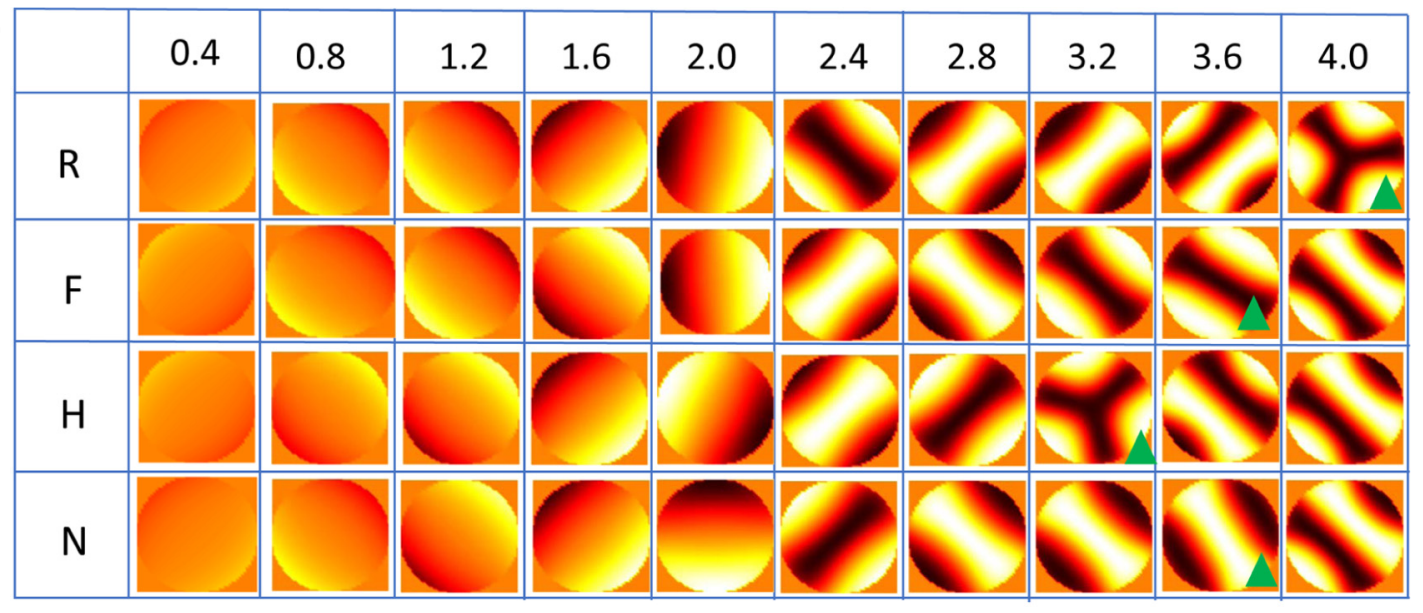

(b)
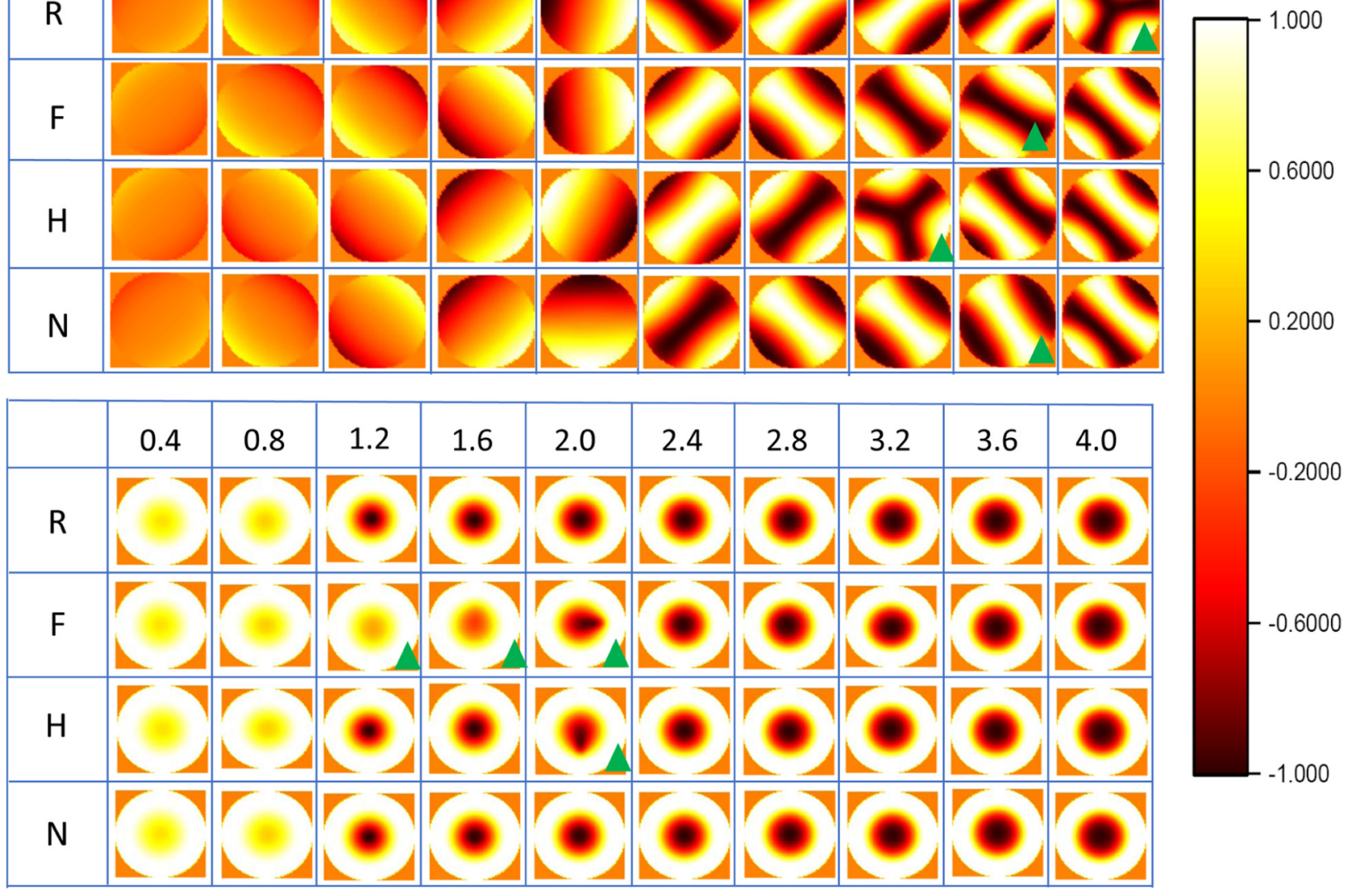

FIG. 2. The profile of the $z$ component of the magnetization of the final states from various initial states (the vertical axis) and for different DMI strengths $D$ in units of $\mathrm{mJ} \mathrm{m}^{-2}$ (the horizontal axis). The four different initial states include random (R), ferromagnetic $(\mathrm{F})$, helix $(\mathrm{H})$, and Néel $(\mathrm{N})$. The results for (a) a disk without pinned spins and (b) a disk with pinned spins.

The size of the unit cell is $1 \mathrm{~nm} \times 1 \mathrm{~nm} \times 1 \mathrm{~nm}$, which is smaller than the exchange length $l_{\mathrm{ex}}=\sqrt{2 A_{\mathrm{ex}} / \mu_{0} M_{s}^{2}} \sim$ $4.17 \mathrm{~nm}$. In our simulations, unless otherwise specified, we use the following parameters: $A_{\mathrm{ex}}=10 \mathrm{pJ} \mathrm{m}^{-1}, M_{s}=$ $9.56 \times 10^{5} \mathrm{~A} \mathrm{~m}^{-1}$, and $K_{u}=0.4 \mathrm{MJ} \mathrm{m}^{-3}$ [32]. The diameter of the nanodisk is set to $50 \mathrm{~nm}$ and the width of the pinning layer is $10 \mathrm{~nm}$. Moreover, the spin direction in the pinned region is chosen to be the positive $z$ direction.

In our system, the DMI originates from spin-orbit coupling at the interface between the metal and the ferromagnet and the vector $\mathbf{D}_{i j}$ lies in the plane of the interface. For an individual skyrmion in an ultrathin nanodisk of thickness $d$ and radius $R$, we may estimate the DMI energy by $2 \pi^{2} D R d$. When a skyrmionic state is present, the demagnetization energy can be estimated by $-2 \pi \mu_{0} M_{s}^{2} W R d$, for a given skyrmion wall width $W=\pi d / 4 K_{u}$ [45]. It turns out that these two energy contributions are of the same order of magnitude, i.e., approximately $10^{-18} \mathrm{~J}$.

To ensure that the final state has the lowest energy, we consider four initial magnetization configurations, namely, ferromagnetic $(\mathrm{F})$, helical $(\mathrm{H})$, random orientation $(\mathrm{R})$, and Néel skyrmion $(\mathrm{N})$. At zero temperature, the initial state evolves according to the LLG equation. We note that the relaxation time - the time the system takes to evolve from an initial state to a final one - is dependent on the choice of the initial state. For example, for a ferromagnetic initial state with $D=4.0 \mathrm{~mJ} \mathrm{~m}{ }^{-2}$, the relaxation time is around $1000 \mathrm{~ns}$, which is roughly 10 times longer than the relaxation times of the other initial states.

\section{NUMERICAL RESULTS}

The main results of this paper are summarized in Fig. 2, which shows the $z$ component of the magnetization of the final states as a function of the DMI strength for different initial spin configurations. Figure 2(a) is for the free boundary condition and Fig. 2(b) is for the fixed boundary condition. This result shows that, with a weak DMI and the free-spins boundary condition, the final state depends largely on the particular choice of the initial state. We 
attribute this to a possible large energy barrier between different final states or local minima that may exist in the energy spectrum. In the case of free spins, for $D \leq$ $2.8 \mathrm{~mJ} \mathrm{~m}^{-2}$, all final states are degenerate in energy and spin configuration (taking into account the in-plane symmetry of the disk), irrespective of the initial state [see also Fig. 3(a)]. For $D>2.8 \mathrm{~mJ} \mathrm{~m}^{-2}$, the initial states evolve into different final states. With a strong DMI, a multidomain state with a Y-shaped strip emerges. We use a green triangle to label, under the same $D$, the final state that, relative to the others, has the highest energy.

In the case of pinned spins, shown in Fig. 2(b), the picture is reversed. For a sufficiently strong DMI $(D \geq$ $2.4 \mathrm{~mJ} \mathrm{~m}^{-2}$ ), the system evolves into a skyrmionic state regardless of the initial state [see also Fig. 3(b)]. Yet with a weak DMI $\left(D<2.0 \mathrm{~mJ} \mathrm{~m}^{-2}\right)$, as Fig. 3(b) shows, different initial states evolve into different final states. We attribute this to the discontinuity in the anisotropy energy distribution imposed by the fixed-spins boundary condition.

\section{A. Stabilization of skyrmions}

To understand how the pinned spins at the edge of the nanodisk helps stabilize skyrmions, we calculate the
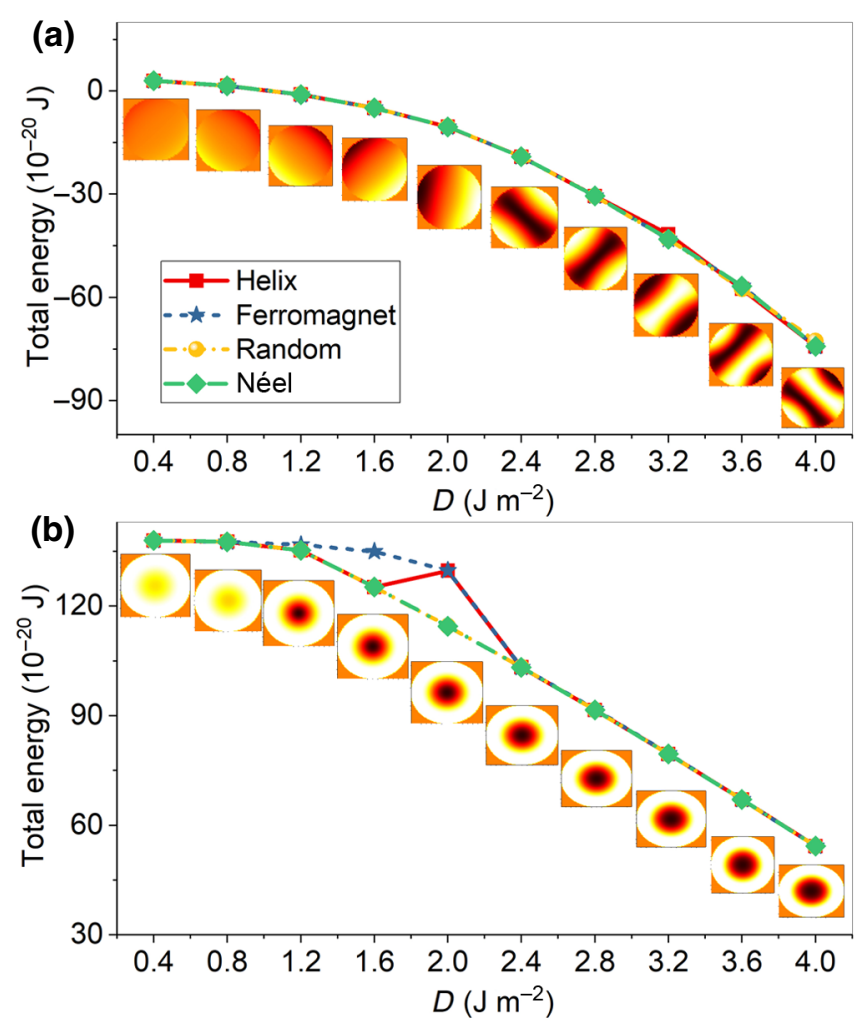

FIG. 3. The total energy and the corresponding $z$ component of the magnetization of the final states as a function of the DMI strength for different initial states. For a disk with a diameter of $50 \mathrm{~nm}$, (a) free spins at the edge and (b) pinned spins at the edges with a diameter of $20 \mathrm{~nm}$. energy of different final states as a function of $D$ with and without the pinning layer. The results are summarized in Fig. 3, in which we only show the magnetization configurations of the final states with the lowest energy.

We show in Fig. 3(a) the total energy as a function of $D$ in the case of free spins. As anticipated, the total energy decreases with $D$. For $D<1.2 \mathrm{~mJ} \mathrm{~m}^{-2}$, the ferromagnetic state has the lowest energy. This agrees with earlier report [46]. As $D$ increases, a multidomain or helical state with a period that is proportional to $A_{\mathrm{ex}} / D$ becomes more stable. Even at a strong DMI, the multidomain or helical state is energetically more favorable than the skyrmionic state, due to the absence of curvature energy [26]. In this case, an instantaneous skyrmionic state can be created by relaxing a skyrmionic initial state with polarity $p=-1$ for about 10 ns. This is illustrated in Fig. 4(a), which shows the spatial profile of $m_{z}$ along the diameter of the nanodisk. This demonstrates that as $D$ increases, the period of the spin helix is shortened and the range of $m_{z}$ extends to cover from -1 to 1 , while the final state evolves continuously from a vortex to a instantaneous skyrmion. This indicates, in the absence of magnetic field and with a free boundary, that nonskyrmionic states are favored.

We plot in Fig. 3(b) the total magnetic energy as a function of $D$ for a nanodisk with a diameter of $70 \mathrm{~nm}$. The nanodisk now consists of a central region that is 50 $\mathrm{nm}$ in diameter (region I) and 20-nm ring-shaped pinning spins (region II), as sketched in Fig. 1(c). Other parameters remain the same as in Fig. 3(a). With $D<1.2 \mathrm{~mJ} \mathrm{~m}^{-2}$, a ferromagnetic state has the lowest energy; while for $D \geq$ $1.2 \mathrm{~mJ} \mathrm{~m}^{-2}$, the lowest energy state becomes skyrmionic. To explain why a skyrmionic state is favored in the presence of pinned spins and a strong DMI, we note that for a given $D$, the spin configuration required to form the DM-driven spin helical state is disrupted (near the edge) and the spin order is suppressed. As a result, the helical state - compared to a ferromagnetic or skyrmionic state - is no longer energetically favorable. In order to retain the helical state as the ground state, as Bogdanov et al. pointed out [9], a sizable $D$ and a weak anisotropy $K_{u}$ are preferred, such that the condition

$$
\sqrt{\frac{A_{\mathrm{ex}} K_{u}}{D}\left(1+\frac{M_{s}^{2}}{2 \mu_{0} K_{u}}\right)}<\frac{\pi}{4}
$$

is satisfied. In the present system, by coercing the spin orientation to $z$ at the edge, we effectively enhance the perpendicular anisotropy. Then the condition to accommodate a helical state as the stable state - i.e., Eq. (4) - no longer holds. We may estimate, using Eq. (4), the threshold value of $D$ as $D_{t}=4 \sqrt{A_{\mathrm{ex}} K_{u}} / \pi \sim 1.7 \mathrm{~mJ} \mathrm{~m}^{-2}$ [47], which is in good agreement with the $1.2 \mathrm{~mJ} / \mathrm{m}^{2}$ found in our simulation.

The spatial profile of the $z$ component of the magnetization provides us with further insight into the effect of 


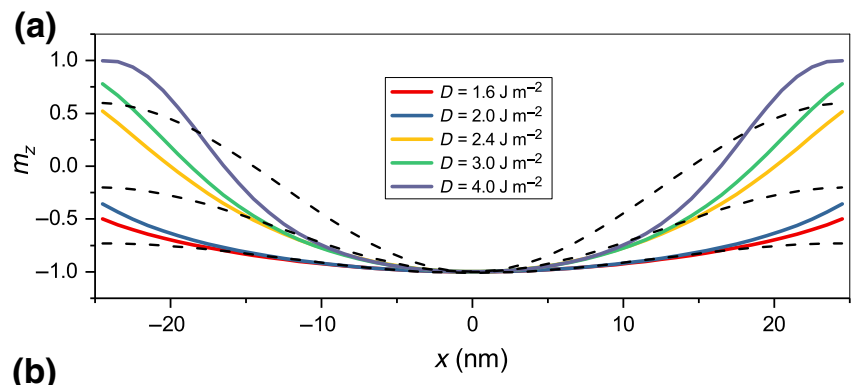

(b)
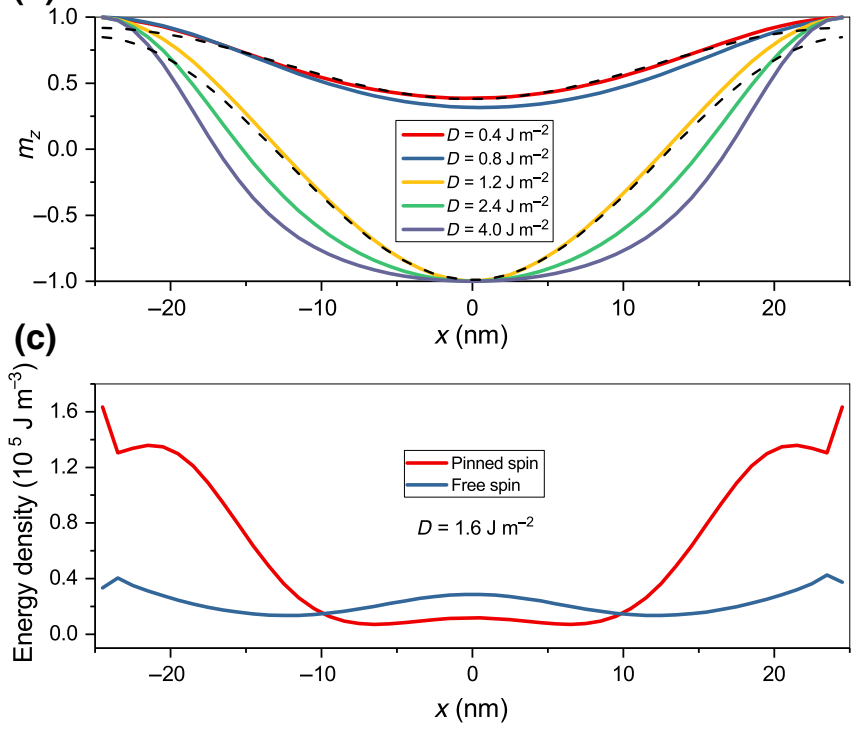

FIG. 4. (a) The spatial profile of the magnetization ( $z$ component) across the core of an instantaneous skyrmion in a disk without a pinning layer. (b) The profile of the magnetization ( $z$ component) as a function of position across the skyrmionic core in a disk with a pinning layer. The dashed line is a cosine function. (c) The local energy density as a function of the position along the disk diameter in the absence (blue) and in the presence (red) of a pinning layer.

the spin boundary condition on the stabilization of the skyrmionic state, as plotted in Fig. 4 . With respect to $D, m_{z}$ exhibits two qualitatively distinct regimes. Under a weak $D$, the stable final state is ferromagnetic, with the spin texture of a magnetic droplet [48]; the total $m_{z}$ in this case is finite. As $D$ increases above a threshold $\left(1.2 \mathrm{~mJ} \mathrm{~m}^{-2}\right)$, the system evolves into a skyrmionic state as the total $m_{z}$ vanishes. In the vicinity of the core, $m_{z}$ is well approximated by a cosine function. At the edges for weak $D$, small deviation appears. As $D$ increases, $m_{z}$ displays a nonlinear transition and it deviates further from the cosine function [see the dashed lines in Fig. 4(b)]. We attribute this behavior to the coupling between the boundary condition and the DMI [49].

In Fig. 4(c), we plot the spatial profile of total energy density across the skyrmionic core. For free spins (blue), the local energy density at the edge is lowered due to the DMI. Near the edge, this creates a weak energy barrier that limits the expansion of the vortex. Such a constraint

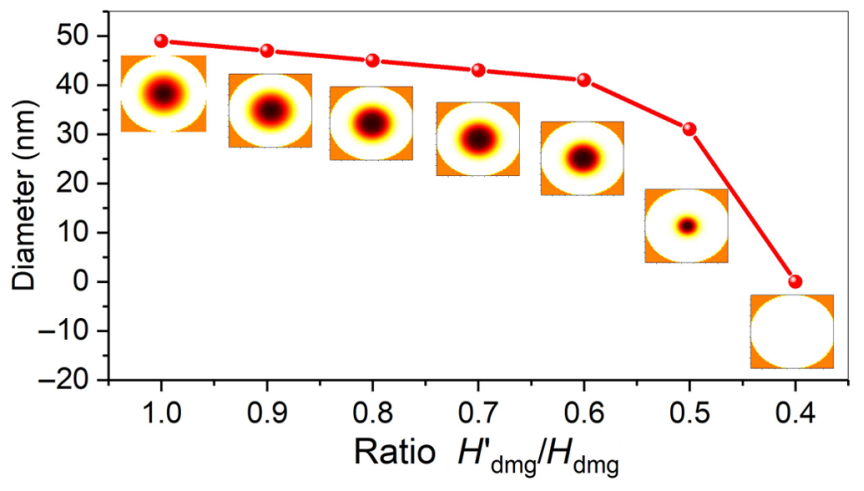

FIG. 5. The diameter of a skyrmion as a function of the ratio of the demagnetization field $\left(H^{\prime}{ }_{\mathrm{dmg}}\right)$ to the original demagnetization field $\left(H_{\mathrm{dmg}}\right)$. The inset shows the $z$ component of the magnetization of the skyrmionic state.

is rather weak, which renders the skyrmionic state instantaneous. With pinned spins (red curve), the energy density near the edge is much higher than it is at the center. In this case, the skyrmion core exerting an outward force toward the edge is suppressed by a high energy barrier. Therefore, the system with locally pinned spins can accommodate a stable skyrmionic state.

\section{B. Effect of the demagnetizing field}

In order to investigate the role of the demagnetizing field in the stabilization of skyrmions, we slowly decrease the strength of the demagnetizing field and study its impact on the skyrmion size, the spatial profile of the magnetostatic energy, and the $z$ component of the magnetization. We recall that in nonhomogeneous textures such as a onedimensional vortexlike state, the demagnetizing field is often considered as a positive correction to the magnetic anisotropy [50]. As a result, an increase in the demagnetizing field leads to a higher magnetic anisotropy and a larger vortex size. In a nanodisk of finite size, however, the size of the skyrmion is proportional to $D / K_{u}$ and hence we expect it to decrease with an increase in $K_{u}$. In Fig. 5(a), we see that for $D=2.4 \mathrm{~mJ} / \mathrm{m}^{2}$, the size of the skyrmion actually decreases with a decrease in the demagnetizing field. This suggests that, in our system, the demagnetizing field is more than just a positive correction to the magnetic anisotropy. In fact, we argue that the demagnetizing field can be interpreted as a positive contribution to the DMI and is thus stabilizing the skyrmion [51,52]. Our simulation supports this claim. It shows that when the strength of the demagnetizing field drops below $40 \%$ of its original value, the skyrmionic state disappears and a ferromagnetic state is favored. Moreover, using the parameters adopted in the simulations, we estimate the demagnetization energy to be about twice as large as the DMI. For a given exchange interaction and $D=2.4 \mathrm{~mJ} / \mathrm{m}^{2}$, taking into account the demagnetizing field, we obtain an effective DMI strength 


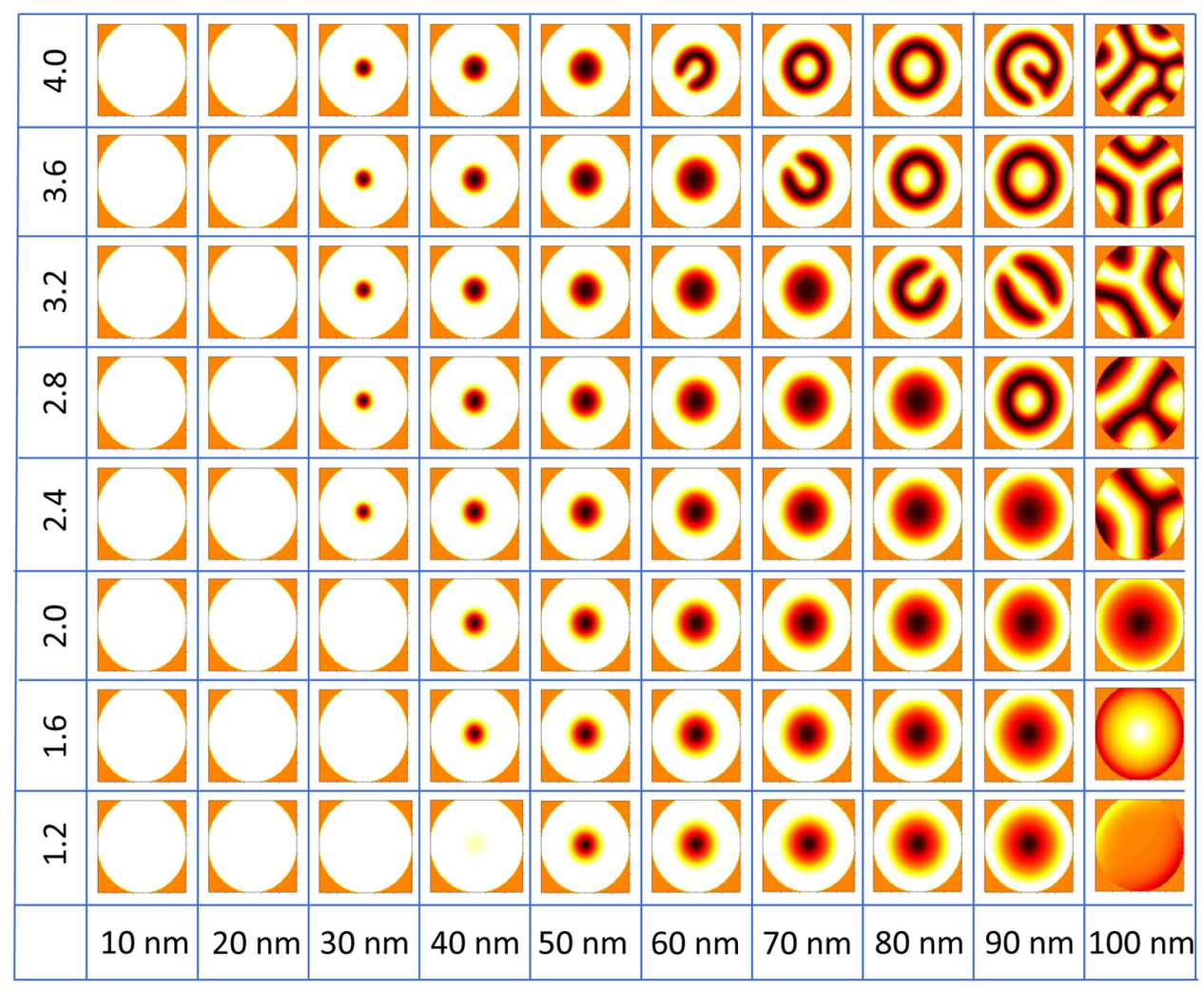

FIG. 6. The magnetization profile of the final state versus the diameter of the unpinned nanodisk (along the horizontal axis) at various values of $D$ (along the vertical axis). The nanodisk diameter $d$ is fixed at $100 \mathrm{~nm}$ and the unpinned region varies from 10 to $100 \mathrm{~nm}$.

$D_{\text {eff }}=7.2 \mathrm{~mJ} / \mathrm{m}^{2}$. (Note that the threshold value to create skyrmionic states is about $D_{\text {eff }}=5.1 \mathrm{~mJ} / \mathrm{m}^{2}$.) As the demagnetizing field drops below $40 \%$, the corresponding effective DMI is lowered to $D_{\text {eff }}=4.3 \mathrm{~mJ} / \mathrm{m}^{2}$, which is below the threshold required to stabilize a skyrmionic state. This analysis is consistent with Fig. 3(b).

\section{Size effect}

In our system, the size of the nanodisk also affects the final state. We show in Fig. 6 the spin texture of the final state as a function of the disk diameter $(d)$ and $D$. As long as the diameter is below $20 \mathrm{~nm}$, the stable state is ferromagnetic regardless of the value of $D$. Under such an extreme confinement, the DMI alone is insufficient to generate a nonuniform state. As the diameter becomes larger, the stable final state evolves into skyrmions at a large DMI. Interestingly, it turns out that the diameter of the skyrmions is as small as $30 \mathrm{~nm}$, yet the helical period is about $50 \mathrm{~nm}$. We attribute this to the fact that the pinned spins support skyrmions and suppress the spin spiral. A further increase in the diameter results in skyrmion-helicity reversal at $D=3.6 \mathrm{~mJ} \mathrm{~m}^{-2}$ and $d=80 \mathrm{~nm}$. A similar phenomenon has also been observed in a two-dimensional system with a strong DMI [53]. Moreover, a biskyrmionic state can be nucleated at $D=3.2 \mathrm{~mJ} \mathrm{~m}^{-2}$ and $d=90 \mathrm{~nm}$ [54]. Finally, with $d=100 \mathrm{~nm}$ and pinning removed, the stable final state of the system is either helix states or a vortex, instead of skyrmions, implying that the pinned spins are instrumental in the nucleation of skyrmions. To conclude this section, we emphasize that, even though all simulations are performed at zero temperature, our central claims remain valid at room temperature.

\section{CONCLUSION}

In a multilayered metallic nanodisk with locally pinned spins, we study the nucleation of magnetic skyrmions in the absence of a magnetic field using micromagnetic simulation. By imposing spin boundary conditions and creating edge potentials at the disk edge, the locally pinned spins ensure stable skyrmionic states. The diameter of the skyrmions is as small as $30 \mathrm{~nm}$ and can be adjusted by changing the DMI strength and the disk size. The creation or annihilation of skyrmions can be controlled by the perpendicular magnetic anisotropy, which is tunable by a bias voltage. Our study opens an alternative avenue for the generation and manipulation of skyrmions in nanostructures.

\section{ACKNOWLEDGMENTS}

H.L. acknowledges support from HeNan University (Grant No. CJ3050A0240050) and the National Natural Science Foundation of China (Grant No. 11804078). C.A.A. acknowledges financial support from the Grant-inAid for Exploratory Research (Grant No. 16K13853), from the Grant-in-Aid for Scientific Research (B) (Grant No. 
17H02929), and from the Japan Society for the Promotion of Science (JSPS). P.Y. acknowledges support from the National Natural Science Foundation of China (Grant No. 11604041), the National Key Research Development Program (under Contract No. 2016YFA0300801), and the National Thousand-Young-Talents Program of China. Y.X.W. acknowledges support from the National Natural Science Foundation of China (Grant No. 11674083). Z.X.C. appreciates the support from the Australian Research Council (Grant No. DP190100150).

[1] G. Dresselhaus, Spin-orbit coupling effects in zinc blende structures, Phys. Rev. 100, 580 (1955).

[2] E. I. Rashba, Properties of semiconductors with an extremum loop. 1. Cyclotron and combinational resonance in a magnetic field perpendicular to the plane of the loop, Sov. Phys. Solid State 2, 1109 (1960).

[3] L. Liu, C. Pai, Y. Li, H. W. Tseng, D. C. Ralph, and R. A. Buhrman, Spin-torque switching with the giant spin Hall effect of tantalum, Science 336, 555 (2012).

[4] Kh. Zakeri, Y. Zhang, J. Prokop, T.-H. Chuang, N. Sakr, W. X. Tang, J. Kirschner, Asymmetric Spin-Wave Dispersion on Fe(110): Direct Evidence of the Dzyaloshinskii-Moriya Interaction, Phys. Rev. Lett. 104, 137203 (2010).

[5] V. Mourik, K. Zuo, S. M. Frolov, S. R. Plissard, E. P. A. M. Bakkers, and L. P. Kouwenhoven, Signatures of Majorana fermions in hybrid superconductor-semiconductor nanowire devices, Science 336, 1003 (2012).

[6] Y. J. Lin, K. Jiménez-García, and I. B. Spielman, Spinorbit-coupled Bose-Einstein condensates, Nature 471, 83 (2011)

[7] A. N. Bogdanov and D. A. Yablonskii, Thermodynamically stable "vortices" in magnetically ordered crystals. The mixed state of magnets, Zh. Eksp. Teor. Fiz. 95, 178 (1989).

[8] A. Bogdanov and A. Hubert, The properties of isolated magnetic vortices, Phys. Stat. Sol. (b) 186, 527 (1994).

[9] A. Bogdanov and A. Hubert, Thermodynamically stable magnetic vortex states in magnetic crystals, JMMM 138, 255 (1994).

[10] I. Dzyaloshinskii, A thermodynamic theory of "weak" ferromagnetism of antiferromagnetics, J. Phys. Chem. Solids 4, 241 (1958).

[11] T. Moriya, Anisotropic superexchange interaction and weak ferromagnetism, Phys. Rev. 120, 91 (1960).

[12] S. Emori, U. Bauer, A.-M. Ahn, E. Martinez, and G. S. D. Beach, Current-driven dynamics of chiral ferromagnetic domain walls, Nat. Mater. 12, 611 (2013).

[13] U. K. Rößler, A. N. Bogdanov, and C. Pfleiderer, Spontaneous skyrmion ground states in magnetic metals, Nature 442, 797 (2006).

[14] A. Thiaville, S. Rohart, É. Jué, V. Cros, and A. Fert, Dynamics of Dzyaloshinskii domain walls in ultrathin magnetic films, Europhys. Lett. 100, 57002 (2010).

[15] X. Z. Yu, Y. Onose, N. Kanazawa, J. H. Park, J. H. Han, Y. Matsui, N. Nagaosa, and Y. Tokura, Real-space observation of a two-dimensional skyrmion crystal, Nature $\mathbf{4 6 5}$, 901 (2010).
[16] S. Mühlbauer, B. Binz, F. Jonietz, C. Pfleiderer, A. Rosch, A. Neubauer, R. Georgii, and P. Böni, Skyrmion lattice in a chiral magnet, Science 323, 915 (2009).

[17] P. Milde, D. Köhler, J. Seidel, L. M. Eng, A. Bauer, A. Chacon, J. Kindervater, S. Mühlbauer, C. Pfleiderer, S. Buhrandt, C. Schütte, and A. Rosch, Unwinding of a skyrmion lattice by magnetic monopoles, Science $\mathbf{3 4 0}$, 1076 (2013).

[18] N. Romming, C. Hanneken, M. Menze, J. E. Bickel, B. Wolter, K. V. Bergmann, A. Kubetzka, and R. Wiesendanger, Writing and deleting single magnetic skyrmions, Science 341, 636 (2013).

[19] P. Hsu, A. Kubetzka, A. Finco, N. Romming, K. Bergmann, and R. Wiesendanger, Electric-field-driven switching of individual magnetic skyrmions, Nat. Nanotechnol. 12, 123 (2017).

[20] J. Iwasaki, M. Mochizuki, and N. Nagaosa, Currentinduced skyrmion dynamics in constricted geometries, Nat. Nanotechnol. 8, 742 (2013).

[21] W. Jiang, P. Upadhyaya, W. Zhang, G. Yu, M. Benjamin Jungfleisch, F. Fradin, J. Pearson, Y. Tserkovnyak, K. Wang, O. Heinonen, S. Velthuis, and A. Hoffmann, Blowing magnetic skyrmion bubbles, Science 349, 283 (2015).

[22] N. Nagaosa and Y. Tokura, Topological properties and dynamics of magnetic skyrmions, Nat. Nanotechnol. 8, 899 (2013).

[23] A. Fert, V. Cros, and J. Sampaio, Skyrmions on the track, Nat. Nanotechnol. 8, 152 (2013).

[24] A. Fert, N. Reyren, and V. Cros, Magnetic skyrmions: Advances in physics and potential applications, Nat. Rev. Mat. 2, 17031 (2017).

[25] M. Uchida, Y. Onose, Y. Matsui, and Y. Tokura, Realspace observation of helical spin order, Science 311, 359 (2006).

[26] S. Rohart and A. Thiaville, Skyrmion confinement in ultrathin film nanostructures in the presence of DzyaloshinskiiMoriya interaction, Phys. Rev. B 88, 184422 (2013).

[27] B. Dupé, G. Bihlmayer, M. Böttcher, S. Bügel, and S. Heinze, Engineering skyrmions in transition-metal multilayers for spintronics, Nat. Commun. 7, 11779 (2016).

[28] F. Zheng, H. Li, S. Wang, D. Song, C. Jin, W. Wei, A. Kovács, J. Zang, M. Tian, Y. Zhang, H. Du, and R. E. Dunin-Borkowski, Direct Imaging of a Zero-Field Target Skyrmion and its Polarity Switch in a Chiral Magnetic Nanodisk, Phys. Rev. Lett. 119, 197205 (2017).

[29] X. Zhao, C. Jin, C. Wang, H. Du, J. Zang, M. Tian, R. Che, and Y. Zhang, Direct imaging of magnetic field-driven transitions of skyrmion cluster states in FeGe nanodisks, Proc. Natl. Acad. Sci. U.S.A. 113, 4918 (2016).

[30] C. Zeng, P. R. C. Kent, M. Varela, M. Eisenbach, G. M. Stocks, M. Torija, J. Shen, and Hanno H. Weitering, Epitaxial Stabilization of Ferromagnetism in the Nanophase of FeGe, Phys. Rev. Lett. 96, 127201 (2006).

[31] S. Woo, K. Litzius, B. Krüger, M. Im, L. Caretta, K. Richter, M. Mann, A. Krone, R. M. Reeve, M. Weigand, P. Agrawal, I. Lemesh, M. Mawass, P. Fischer, M. Kläui, and G. S. D. Beach, Observation of room-temperature magnetic skyrmions and their current-driven dynamics in ultrathin metallic ferromagnets, Nat. Mat. 15, 501 (2016). 
[32] C. Moreau-Luchaire, C. Moutafis, N. Reyren, J. Sampaio, C. A. F. Vaz, N. Van Horne, K. Bouzehouane, K. Garcia, C. Deranlot, P. Warnicke, P. Wohlhüter, J.-M. George, M. Weigand, J. Raabe, V. Cros, and A. Fert, Additive interfacial chiral interaction in multilayers for stabilization of small individual skyrmions at room temperature, Nat Nanotechnol. 11, 444 (2016).

[33] Z. Luo, T. P. Dao, A. Hrabec, J. Vijayakumar, A. Kleibert, M. Baumgartner, E. Kirk, J. Cui, T. Savchenko, G. Krishnaswamy, L. J. Heyderman, and P. Gambardella, Chirally coupled nanomagnets, Science 363, 1435 (2019).

[34] G. Chen, S. Kang, C. Ophus, A. N'Diaye, H. Kwon, R. Qiu, C. Won, K. Liu, Y. Wu, and A. Schmid, Out-of-plane chiral domain wall spin-structures in ultrathin in-plane magnets, Nat. Commun. 8, 15302 (2017).

[35] X. Portier and A. K. Petford-Long, The formation of $360^{\circ}$ domain walls in magnetic tunnel junction elements, Appl. Phys. Lett. 76, 754 (2000).

[36] J. Sayama, T. Asahi, K. Mizutani, and T. Osaka, Newly developed $\mathrm{SmCo}_{5}$ thin film with perpendicular magnetic anisotropy, J. Phys. D Appl. Phys, 37, L1 (2004).

[37] J. F. Herbst, J. J. Croat, F. E. Pinkerton, and W. B. Yelon, Relationships between crystal structure and magnetic properties in $\mathrm{Nd}_{2} \mathrm{Fe}_{14} \mathrm{~B}$, Phys. Rev. B 29, 4176(R) (1984).

[38] X. Li, C. W. Leungb, C. Chiu, K.-W. Lin, Mansun Chan, Y. Zhou, and Philip W. T. Ponga, Exchange bias study of sub$100 \mathrm{~nm}$-diameter $\mathrm{CoFeB} / \mathrm{IrMn}$ antidot and nanodot arrays fabricated by nanosphere lithography, Phys. Lett. A 381, 2709 (2017).

[39] W. F. Brown, Micromagnetics (Interscience Publishers, New York, NY, 1963).

[40] A. Vansteenkiste, J. Leliaert, M. Dvornik, M. Helsen, F. Garcia-Sanchez, and B. Van Waeyenberge, The design and verification of MuMax3, AIP Adv. 4, 107133 (2014).

[41] R. D. McMichael, M. J. Donahue, D. G. Porter, and Jason Eicke, Comparison of magnetostatic field calculation methods on two-dimensional square grids as applied to a micromagnetic standard problem, J. Appl. Phys. 85, 5816 (1999).

[42] C. Shir, Preliminary computations of magnetic distributions in ion-implanted garnet films, IEEE Trans. Magn. 17, 2401 (1981).
[43] A. N. Bogdanov and U. K. Rößler, Chiral Symmetry Breaking in Magnetic Thin Films and Multilayers, Phys. Rev. Lett. 87, 037203 (2001).

[44] J. M. Winter, Bloch wall excitation: Application to nuclear resonance in a Bloch wall, Phys. Rev. 124, 452 (1961).

[45] X. S. Wang, H. Y. Yuan, and X. R. Wang, A theory on skyrmion size, Commun. Phys. 1, 31 (2018).

[46] J. Sampaio, V. Cros, S. Rohart, A. Thiaville, and A. Fert, Nucleation, stability and current-induced motion of isolated magnetic skyrmions in nanostructures, Nat. Nanotechnol. 8, 839 (2013).

[47] A. B. Butenko, A. A. Leonov, A. N. Bogdanov, and U. K. Rößler, Theory of vortex states in magnetic nanodisks with induced Dzyaloshinskii-Moriya interactions, Phys. Rev. B 80, 134410 (2009).

[48] Ferran Macia, Dirk Backes, and Andrew D. Kent, Stable magnetic droplet solitons in spin-transfer nanocontact, Nat. Nanotechnol. 9, 992 (2013).

[49] F. Garcia-Sanchez, Pablo Borys, Arne Vansteenkiste, JooVon Kim, and Robert L. Stamps, Nonreciprocal spin-wave channeling along textures driven by the DzyaloshinskiiMoriya interaction, Phys. Rev. B 89, 224408 (2014).

[50] A. Bogdanov and A. Hubert, The stability of vortex-like structures in uniaxial ferromagnets, J. MMM 195, 182 (1999).

[51] M. Ezawa, Giant Skyrmions Stabilized by Dipole-Dipole Interactions in Thin Ferromagnetic Films, Phys. Rev. Lett. 105, 197202 (2010).

[52] F. Blüttner, I. Lemesh, and G. S. D. Beach, Theory of isolated magnetic skyrmions: From fundamentals to room temperature applications, Sci. Rep. 8, 4464 (2018).

[53] W. Jiang, S. Zhang, X. Wang, C. Phatak, Q. Wang, W. Zhang, M. Benjamin Jungfleisch, J. E. Pearson, Y. Liu, J. Zang, X. Cheng, A. Petford-Long, A. Hoffmann, and S. G. E. te Velthuis, Quantifying chiral exchange interaction for Néel-type skyrmions via Lorentz transmission electron microscopy, Phys. Rev. B 99, 104402 (2019).

[54] X. Li, S. Zhang, H. Li, D. Alba Venero, J. S White, R. Cubitt, Q. Huang, J. Chen, L. He, G. van der Laan, W. Wang, T. Hesjedal, and F. Wang, Oriented 3D magnetic biskyrmions in MnNiGa bulk crystals, Adv. Mat. 31, 1900264 (2019). 